\title{
Grey Sets and Greyness
}

\author{
Yingjie Yang*, Robert John \\ Centre for Computational Intelligence, School of Computing, De Montfort University, The \\ Gateway, Leicester, LE1 9BH, England
}

\begin{abstract}
This paper discusses the application of grey numbers for uncertainty representation. It highlights the difference between grey sets and interval-valued fuzzy sets, and investigates the degree of greyness for grey sets. It facilitates the representation of uncertainty not only for elements of a set, but also the set itself as a whole. Our results show that a grey set could be specified for interval-valued fuzzy sets or rough sets under special conditions. With the notion of grey sets and their associated degrees of greyness, various set operations between grey sets are discussed.
\end{abstract}

Keywords: Grey sets, Fuzzy sets, Rough sets

\section{Introduction}

The information contained in a real-world database is usually incomplete and vague. Non-deterministic information systems [12, 24, 29, 31] are developed to deal with incomplete information, and fuzzy sets [56] are applied to describe vague information. However, non-deterministic information systems and fuzzy systems adopt completely different representations and are usually applied separately from each other. There have been some efforts to combine the two different uncertainties in one model, such as the non-deterministic fuzzy classification systems [11] and interval-valued fuzzy sets [38]. However, the nondeterministic fuzzy classification systems consider the non-deterministic feature

\footnotetext{
* Corresponding author

Email addresses: yyang@dmu.ac.uk (Yingjie Yang), rij@dmu.ac.uk (Robert John)
} 
only in aggregation operators, and interval-valued fuzzy sets can not represent limited options of membership values in a discrete set. Therefore, a systematic representation of both vagueness (fuzziness) and incompleteness appears attractive.

Grey systems have emerged as an effective model for systems with partial information $[14,23,25,26]$. They provide an alternative for representing uncertainty in systems in addition to the mainstream models like fuzzy sets and rough sets. Grey sets apply the basic concepts of grey numbers in grey systems, and consider the characteristic function values of a set as grey numbers. If we restrict characteristic function values within $[0,1]$, grey sets can be considered as an extension to fuzzy sets. Grey numbers and intervals have some similarity and grey sets are considered to be the same as interval-valued fuzzy sets [15]. With the increasing applications of grey systems, the combination of grey sets with fuzzy sets and rough sets have been investigated recently [40,42]. However, all these research works considered grey sets using interval grey numbers only, and such a restriction makes grey sets and interval-valued fuzzy sets equivalent. Without understanding the difference between grey sets and interval-valued fuzzy sets, grey sets would appear as simply a different name for interval-valued fuzzy sets. In fact, a grey number has some special features which are not shared by intervals, and the same applies to grey sets. Compared with interval-valued fuzzy sets, grey sets provide better coverage for partial information dealt with by non-deterministic information systems. Obviously, it is necessary to give a clear definition of a grey set and investigate their differences to other extensions of fuzzy sets. The degree of greyness of a grey number is another important feature of grey numbers different from intervals, and the degree of greyness of a grey set has not been investigated sufficiently to date. Therefore, this paper focuses mainly on the difference between grey numbers and intervals, grey sets and interval-valued fuzzy sets and the degree of greyness of a grey set in set operations.

The paper is structured as follows. In the next Section a brief overview of fuzzy sets, rough sets and grey numbers is provided. Section 3 extends the 
definition of grey numbers to cover wider situations and defines a new whitenisation function for grey numbers. Section 4 then defines grey sets and proves their properties in relation to interval-valued fuzzy sets and rough sets. Section 5 discusses various operations on grey sets and the corresponding degrees of greyness. Finally, in Section 6 we draw out the conclusions.

\section{Preliminaries}

We first define some relevant concepts.

Definition 1 (Fuzzy sets [56]). Let $U$ denote a universe of discourse. Then a fuzzy set $A$ in $U$ is defined as a set of ordered pairs

$$
A=\left\{\left\langle x, \mu_{A}(x)\right\rangle: x \in U\right\}
$$

where $\mu_{A}: U \longrightarrow[0,1]$ is the membership function of $A$ and $\mu_{A}(x)$ is the grade of belongingness of $x$ into $A$.

The membership function value [21] can be any real number between 0 and 1 which implies a fuzzy concept or graded set boundary [19].

A fuzzy set with interval values as its membership values is called an intervalvalued fuzzy set.

Definition 2 (Interval-valued fuzzy sets [38]). Let $D[0,1]$ be the set of all closed subintervals of the interval $[0,1]$. $U$ is the universe of discourse, $x$ is an element and $x \in U$. An interval-valued fuzzy set in $U$ is given by set $A$

$$
A=\left\{\left\langle x, M_{A}(x)\right\rangle: x \in U\right\}
$$

with $M_{A}: U \rightarrow D[0,1]$.

The membership of an individual element is thus reflected by an interval instead of a single value. An intuitionistic fuzzy set [2] is mathematically equivalent to an interval-valued fuzzy set although some semantic differences still exist $[4-6,9,15,17,39]$. 
Definition 3 (Intuitionistic fuzzy sets [2]). An intuitionistic fuzzy set $A$ in $U$ is given by

$$
A=\left\{\left\langle x, \mu_{A}(x), \nu_{A}(x)\right\rangle \mid x \in U\right\}
$$

where

$$
\mu_{A}: U \rightarrow[0,1], \nu_{A}: U \rightarrow[0,1]
$$

and

$$
0 \leq \mu_{A}(x)+\nu_{A}(x) \leq 1 \quad \forall x \in U .
$$

For each $x$, the numbers $\mu_{A}(x)$ and $\nu_{A}(x)$ are the degree of membership and degree of non-membership of $x$ to $A$ respectively.

Type-2 fuzzy sets allow membership grades that are type- 1 fuzzy sets. These are sometimes referred to as "fuzzy-fuzzy" [10,27]. This is a general extension to fuzzy sets, and interval-valued fuzzy sets can be considered as a special case of type-2 fuzzy sets.

Definition 4 (Type-2 fuzzy sets [27]). A type-2 fuzzy set $A$ is characterized by a type- 2 membership function $\mu_{A}(x, u)$, where $x \in U$ and $u \in J_{x} \subseteq[0,1]$, i.e.,

$$
A=\left\{\left\langle(x, u), \mu_{A}(x, u)\right\rangle \mid \forall x \in U, \forall u \in J_{x} \subseteq[0,1]\right\}
$$

in which $0 \leq \mu_{A}(x, u) \leq 1$. $A$ can also be expressed as

$$
A=\int_{x \in U} \int_{u \in J_{x}} \mu_{A}(x, u) /(x, u) \quad J_{x} \subseteq[0,1]
$$

where $\iint$ denotes union over all admissible $x$ and $u$. For discrete universes of discourse $\int$ is replaced by $\sum$.

Independent from fuzzy sets, a non-deterministic information system is applied to represent incomplete information.

Definition 5 (Non-deterministic information systems [29]). A non-deterministic information system is a quadruplet $\left(O B, A T,\left\{V A L_{a} \mid a \in A T\right\}, g\right)$, where $O B$ is a finite set whose elements are called objects, $A T$ is a finite set whose elements 
are called attributes, $V A L_{a}$ is a finite set whose elements are called attribute values, $g$ is a mapping from $O B \times A T$ to a power set of $\cup_{a \in A T} V A L_{a}$, i.e., $g: O B \times A T \rightarrow P\left(\cup_{a \in A T} V A L_{a}\right) . g(x, a)$ is interpreted as if there is an actual value in this set but it is not known.

Obviously, non-deterministic information systems do not employ fuzzy memberships and hence it cannot reveal fuzziness. In the same time, $g(x, a)$ could be represented by a finite set which is not representable by interval values in the interval-valued fuzzy sets.

Another model for uncertainty is rough sets. Rough sets were put forward firstly by Pawlak in 1982 [30]. Rough set theory does not directly consider the uncertain status of individual elements, but it focuses on the approximate sets. When a set $A$ can not be described in a precise way the lower and upper approximations are used instead.

There are many different interpretations of the notion of rough sets $[3,20$, $32,41,51,53,55,57]$. Similar to [28], we adopt the set-oriented interpretation of rough sets $[20,28,30,32,36,46,47,51,52]$ and define a rough set as a pair of definable sets.

Definition 6 (Rough sets $[\mathbf{3 2}, \mathbf{5 1}])$. Let pair apr $=(U, B)$ be an approximation space on $U$ and $U / B$ denote the set of all equivalence classes of $B$. $B$ is an equivalence relation on $U$. A set which is a union of the empty set $\emptyset$ and elements of $U / B$ is called a definable set. The family of all definable sets in approximation space apr is denoted by $\operatorname{Def}(a p r)$. Given two subsets $\underline{A}, \bar{A} \in \operatorname{Def}(a p r)$ with $\underline{A} \subseteq \bar{A}$, the pair $(\underline{A}, \bar{A})$ is called a rough set.

The pair $(\underline{A}, \bar{A})$ approximates a set $A$, and $\underline{A} \subseteq A \subseteq \bar{A}$. $\underline{A}$ is the lower approximation of $A$, and $\bar{A}$ is the upper approximation of $A$. The significance of uncertain elements is measured by the roughness of a rough set.

Definition 7 (Roughness of approximation [32]). The roughness $R^{\circ}(A)$ for a set $A$ approximated by $(\underline{A}, \bar{A})$ is defined as the significance of the un- 
certain elements to the set.

$$
R^{\circ}(A)=\frac{|\bar{A}-\underline{A}|}{|\bar{A}|}
$$

Rough sets and fuzzy sets are two different theories capturing two distinct aspects of imperfection in knowledge: indiscernibility and vagueness [18]. However, as a concept induced from fuzzy sets, shadowed sets [35] have a close relationship with rough sets as well. Considering a fuzzy set $A \in \mathbb{U}$, we elevate those membership values that are high enough to 1 and reduce those substantially low membership values to 0 , and represent those values in between as $[0,1]$, then we have transformed the fuzzy set to a shadowed set [35].

$$
A: \mathbb{U} \longrightarrow\{0,1,[0,1]\}
$$

Here, each element $x$ is associated with 0,1 or $[0,1]$. The elements for which $A(x)$ attains 1 constitute its core, and the elements where $A(x)=[0,1]$ form a shadow where uncertainty exists. Shadowed sets do not require precise membership values, and partition the elements of a fuzzy set into three categories: Yes (1), No $(0)$ and Unknown $([0,1])$. In this sense, shadowed sets are conceptually close to rough sets.

A rough set approximates a subset using two definable sets, and its representation actually forms a set of subsets. Generalising this idea, we get a more generalised set: interval sets.

Definition 8 (Interval sets $[\mathbf{5 0}, \mathbf{5 4}]$ ). Let $U$ be the finite universe, and $2^{U}$ be its power set. A subset of $2^{U}$ of the form

$$
A=\left[A_{1}, A_{2}\right]=\left\{A \in 2^{U} \mid A_{1} \subseteq A \subseteq A_{2}\right\}
$$

is called a closed interval set, where it is assumed $A_{1} \subseteq A_{2}$.

Obviously, the rough set model might be interpreted as a special case of interval sets. 
As a different model for uncertainty representation, grey systems were proposed by Professor Julong Deng in 1982 [13]. In grey systems, the information is classified into three categories: white with completely certain information, grey with insufficient information, and black with totally unknown information. Grey numbers are the basic concepts in grey systems.

Definition 9 (Grey numbers $[\mathbf{1 3}, \mathbf{2 5}]$ ). A grey number is a number with clear upper and lower boundaries but which has an unknown position within the boundaries.

A grey number for the system is expressed mathematically as [7]

$$
g^{ \pm} \in\left[g^{-}, g^{+}\right]=\left\{g^{-} \leq t \leq g^{+}\right\}
$$

where $g^{ \pm}$is a grey number, $t$ is information, $g^{-}$and $g^{+}$are the upper and lower limits of the information. The arithmetic of grey numbers is very similar to interval values $[1]$.

Definition 10 (Degree of greyness of a grey number $[13,25])$. The significance of the interval to the unknown number represented by a grey number is called the degree of greyness.

It can be expressed as

$$
g^{\circ}\left(g^{ \pm}\right)=f\left(g^{-}, g^{+}\right)
$$

Here, $f$ is a function to determine the significance of the interval to $g^{ \pm}$. Let $D=\left[d_{\min }, d_{\max }\right]$ be the domain of values represented by a grey number $g^{ \pm} \in\left[g^{-}, g^{+}\right]$, then we have $d_{\min } \leq g^{-}, g^{+} \leq d_{\max }$ and $f\left(g^{-}, g^{+}\right)=\frac{g^{+}-g^{-}}{d_{\max }-d_{\min }}$. There are two special situations for $g^{ \pm}$:

- If $g^{-}=g^{+}$, then we call $g^{ \pm}$a white number

- If $g^{-}=d_{\min }$ and $g^{+}=d_{\max }$, then we call $g^{ \pm}$a black number 
Obviously, a white number is a single crisp value where we have full knowledge. On the contrary, a black number is a grey number we know nothing about it.

It should be noted that grey numbers could be discrete when the candidate values are finite [25]. For example, if a grey number can only be one value among the integers 1, 2, 3, 4 and 5, it would be represented as:

$$
g^{ \pm} \in\{1,2,3,4,5\}
$$

Both traditional crisp sets and fuzzy sets need a clearly defined membership or characteristic function value. Rough sets have a rough membership function representing the probability of an element being a member of the set [22]. However, this clearly defined number is difficult to know in certain situations. This raises the question of how to determine this crisp membership value: how to determine such a crisp value for a fuzzy element? Interval-valued/Intuitionistics fuzzy sets have successfully expressed this situation in the case of fuzzy sets. For epistemic uncertainty, an interval representation means that any value within the interval is a possible value. However, we may know that the possible value can only be one of a finite number of values within the interval. For this situation, an interval representation cannot help.

Type-2 fuzzy sets tackle the difficulty of determining a membership value by replacing it with another fuzzy set. This helps but there is still an issue with the secondary grade. This problem comes from the circle of explaining one fuzzy set with another fuzzy set. The uncertain membership value needs a representation that can express both the possible values and the fact that it is a single value as defined in Definition 1. It should consider both the continuous and discrete situation as well.

Obviously, grey numbers provide us with a convenient tool to represent this membership value with incomplete information. Due to the adoption of interval representation for grey numbers, however, grey sets have been considered the 
same as interval-valued fuzzy sets $[15,16]$. Some recent publications have reported work on the combination of grey sets with rough sets [40, 42]. However, in their work, grey sets are still defined using interval grey numbers which makes them equivalent to interval-valued fuzzy sets.

Based on our work on grey sets $[45,48]$ and grey numbers $[43,49]$, here we extend our definition of grey sets to include all grey numbers and analyse the relationship of grey sets with interval-valued fuzzy sets and rough sets.

\section{Generalised Grey Numbers}

Before the discussion of grey sets, we extend the definition of grey numbers to include all possible situations. Grey systems refer to partially known systems, and grey numbers denote partially known numbers. In this sense, intervals can be considered as a special case of grey numbers where we know the scope of the underlying number but do not know its exact position inside the continuous scope. However, the candidate of a partial known number could be selected from a finite set of numbers, or a set of intervals. Here, we extend the definition in Definition 9 to give a clear definition of grey numbers.

Definition 11 (Generalised grey numbers). Let $g^{ \pm} \in \Re$ be an unknown real number within a union set of closed or open intervals

$$
g^{ \pm} \in \bigcup_{i=1}^{n}\left[a_{i}^{-}, a_{i}^{+}\right]
$$

$i=1,2, \ldots, n, n$ is an integer and $0<n<\infty, a_{i}^{-}, a_{i}^{+} \in \Re$ and $a_{i-1}^{+} \leq a_{i}^{-} \leq$ $a_{i}^{+} \leq a_{i+1}^{-}$. For any interval $\left[a_{i}^{-}, a_{i}^{+}\right] \subseteq \bigcup_{i=1}^{n}\left[a_{i}^{-}, a_{i}^{+}\right], p_{i}$ is the probability for $g^{ \pm} \in\left[a_{i}^{-}, a_{i}^{+}\right]$. If the following conditions hold

- $p_{i}>0$

- $\sum_{i=1}^{n} p_{i}=1$

then we call $g^{ \pm}$a generalised grey number. $g^{-}=\inf _{a_{i}^{-} \in g^{ \pm}} a_{i}^{-}$and $g^{+}=$ $\sup _{a_{i}^{+} \in g^{ \pm}} a_{i}^{+}$are called as the lower and upper limits of $g^{ \pm}$. 
From this definition, it is impossible for a grey number to have more than one number in its candidate set to be the underlying white number. This is different from a rough sets with probabilistic membership values [32-34].

Hereafter, the term grey numbers is adopted to represent generalised grey numbers. We use the standard interpretation of an interval (a set of real numbers). The union operation refers to standard union of sets. It should be noted that the intervals involved in grey numbers do not need to be closed although our expression uses the closed representation. Obviously, Definition 11 is much more general than Equation (2). It removes the limitation for open sets and discrete sets to represent a grey number. A grey number could be represented as a set of intervals with gaps in between. For example, $g^{ \pm} \in\{[5,6],[10,12]\}$ is a grey number where its underlying white number may get its value from $[5,6]$ or $[10,12]$, but we know for sure that it will not get its value within $(6,10)$. This is clearly different from an interval $[5,12]$ where $(6,10)$ is a valid part for candidate values. In the same time, we could have a grey number with candidate values in a discrete set $g^{ \pm} \in\{5,6,10,12\}$, which is clearly not representable by an interval. From Definition 11, it is also clear that a grey number $g^{ \pm}$is different from the set $\bigcup_{i=1}^{n}\left[a_{i}^{-}, a_{i}^{+}\right]$. The grey number $g^{ \pm}$represents only one number which is not clearly identified among the elements in set $\bigcup_{i=1}^{n}\left[a_{i}^{-}, a_{i}^{+}\right]$.

Similar to interval algebra [50], we can perform arithmetic with grey numbers through the arithmetic operations on their members. Let $a^{ \pm}$and $b^{ \pm}$be two grey numbers, and let $*$ denote an arithmetic operation,,$+- \times$ or $\div$ on pairs of real numbers. The arithmetic operation $*$ between two grey numbers can be expressed as

$$
a^{ \pm} * b^{ \pm}=\left\{x * y \mid x \in \bigcup_{i=1}^{m}\left[a_{i}^{-}, a_{i}^{+}\right], y \in \bigcup_{j=1}^{n}\left[b_{j}^{-}, b_{j}^{+}\right]\right\}
$$


The result of $a^{ \pm} * b^{ \pm}$is still a grey number unless $0 \in \bigcup_{j=1}^{n}\left[b_{j}^{-}, b_{j}^{+}\right]$. It is easy to derive the following formulas for $a^{ \pm} * b^{ \pm}[43]$ :

$$
\begin{gathered}
a^{ \pm}+b^{ \pm}=\bigcup_{i=1}^{m} \bigcup_{j=1}^{n}\left[a_{i}^{-}+b_{j}^{-}, a_{i}^{+}+b_{j}^{+}\right] \\
a^{ \pm}-b^{ \pm}=\bigcup_{i=1}^{m} \bigcup_{j=1}^{n}\left[a_{i}^{-}-b_{j}^{+}, a_{i}^{+}-b_{j}^{-}\right] \\
a^{ \pm} \times b^{ \pm}=\bigcup_{i=1}^{m} \bigcup_{j=1}^{n}\left[\min \left\{a_{i}^{-} b_{j}^{-}, a_{i}^{+} b_{j}^{+}, a_{i}^{-} b_{j}^{+}, a_{i}^{+} b_{j}^{-}\right\}, \max \left\{a_{i}^{-} b_{j}^{-}, a_{i}^{+} b_{j}^{+}, a_{i}^{-} b_{j}^{+}, a_{i}^{+} b_{j}^{-}\right\}\right] \\
a^{ \pm} \div b^{ \pm}=\bigcup_{i=1}^{m} \bigcup_{j=1}^{n}\left[\min \left\{\frac{a_{i}^{-}}{b_{j}^{-}}, \frac{a_{i}^{+}}{b_{j}^{+}}, \frac{a_{i}^{-}}{b_{j}^{+}}, \frac{a_{i}^{+}}{b_{j}^{-}}\right\}, \max \left\{\frac{a_{i}^{-}}{b_{j}^{-}}, \frac{a_{i}^{+}}{b_{j}^{+}}, \frac{a_{i}^{-}}{b_{j}^{+}}, \frac{a_{i}^{+}}{b_{j}^{-}}\right\}\right]
\end{gathered}
$$

Here, we assume $a_{i}^{-} \leq a_{i}^{+}$and $b_{j}^{-} \leq b_{j}^{+}$. For Equation (3), we assume $b_{j}^{-} \neq 0$ and $b_{j}^{+} \neq 0$.

In real world applications, the domain $D$ of a grey number is usually a subset of $\Re$, and the degree of greyness of a grey number can be defined on such a domain.

Definition 12 (Degree of greyness of a grey number). Let $D \subset \Re$ and $g^{ \pm} \in D, d_{\min }, d_{\max } \in \Re$ are the minimum and maximum values of $D$. The degree of greyness of $g^{ \pm}$is defined as

$$
g^{\circ}=\frac{\left|g^{+}-g^{-}\right|}{\left|d_{\max }-d_{\min }\right|}
$$

Obviously, we have

- $g^{\circ}=0$ iff $g^{+}=g^{-}$

- $g^{\circ}=1$ iff $g^{-}=d_{\min }$ and $g^{+}=d_{\max }$

The degree of greyness of a grey number depends only on the two limits of a grey number and has nothing to do with the cardinality of its candidate set. For example, $g_{1}{ }^{ \pm} \in[40,60]$ and $g_{2}{ }^{ \pm} \in\{40,60\}$ are defined on $[0,100]$ and have the same degree of greyness

$$
g_{1}{ }^{\circ}=g_{2}{ }^{\circ}=\frac{60-40}{100-0}=0.2
$$


but their cardinalities are completely different:

$$
\operatorname{Card}\left(g_{1}^{ \pm}\right)=\infty, \quad \operatorname{Card}\left(g_{2}^{ \pm}\right)=2
$$

This indicates that the degree of greyness is a parameter for the grey number rather than any candidate in its candidate set. This is different from the probability for each number in its candidate set to be the underlying white number. In this sense, white numbers and black numbers can be easily defined using the concept of degree of greyness.

Definition 13 (White numbers). For any grey number $g^{ \pm} \in D(D \subset \Re)$, if $g^{\circ}=0$, then this $g^{ \pm}$is called a white number.

Definition 14 (Black numbers). For any grey number $g^{ \pm} \in D(D \subset \Re)$, if $g^{\circ}=1$, then this $g^{ \pm}$is called a black number.

Clearly, a white number is a normal crisp number where everything is clearly known, and a black number is a number with nothing known. For a grey number, we have $0 \leq g^{\circ} \leq 1$. Adding new information, the degree of greyness of a grey number could be reduced. In the traditional theory of grey systems, a whitenisation function is applied to convert a grey number into a white number:

$$
g=f\left(g^{-}, g^{+}\right)
$$

We redefine this operation as a process of reducing uncertainty from a grey number, and its result is a new grey number with a lower degree of greyness.

Definition 15 (Whitenisation of grey numbers). Let $g^{ \pm} \in D(D \subset \Re)$ be a grey number, and $\nabla g^{ \pm}=F\left(g^{ \pm}\right)$be a function defined on $D\left(\nabla g^{ \pm} \in D\right)$. If $\nabla g^{\circ}<g^{\circ}$, then $\nabla g^{ \pm}=F\left(g^{ \pm}\right)$is called a whitenisation function, and $\nabla g^{ \pm}$is called a whitenisation of $g^{ \pm}$.

There are many possible whitenisation functions for a grey number. The whitenisation function satisfying $\nabla g^{\circ}=0$ is called a complete whitenisation 
function. Clearly, the complete whitenisation of a grey number $g^{ \pm}$is a white number $\nabla g$

$$
\nabla g^{ \pm}=\nabla g \text { if } \nabla g^{\circ}=0
$$

The traditional whitenisation function in grey system theory is actually a complete whitenisation function.

With a given value of degree of greyness, a grey number can have different candidate sets. We can further classify grey numbers into different categories according to theorem 1 .

Theorem 1. $g^{ \pm}$is a grey number defined by Definition 11. The following properties hold for $g^{ \pm}$:

- $g^{ \pm}$is a continuous grey number $g^{ \pm} \in\left[a_{1}^{-}, a_{n}^{+}\right]$iff $a_{i}^{-}=a_{i-1}^{+}(\forall i>1)$ or $n=1$

- $g^{ \pm}$is a discrete grey number $g^{ \pm} \in\left\{a_{1}, a_{2}, \ldots, a_{n}\right\}$ iff $a_{i}=a_{i}^{-}=a_{i}^{+}$

- $g^{ \pm}$is a mixed grey number iff part of its intervals shrink to crisp numbers and others keep as intervals.

This theorem is simple and easy to prove, but it reveals a crucial difference between grey numbers and interval values: discrete grey numbers or mixed grey number are possible in addition to continuous grey numbers. A discrete grey number $g^{ \pm}$can be expressed as follow:

$$
g^{ \pm} \in\left\{g^{-}, g_{1}, g_{2}, \ldots, g_{k}, g^{+}\right\}
$$

where, $g^{-} \leq g_{1} \leq g_{2} \leq \ldots \leq g_{k} \leq g^{+}$and $0 \leq k<\infty$.

Example 1. Considering an example in holiday travel. A holiday maker is planning for his one week holiday travel in August. He has to plan his holiday starting from one Monday in August: 6, 13, 20 and 27. The total covered distance is known to be 1000 miles. However, the consumption of petrol could only be evaluated as some value between 90 litres to 120 litres. 
If we consider the date as a number, then we get three numbers in the holiday plan: a discrete grey number for starting date $\{6,13,20,27\}$, a white number for distance 1000 and a continuous grey number for petrol consumption [90, 120]. Although the starting date and petrol consumption are represented as sets, they are actually two numbers rather than sets. $\{6,13,20,27\}$ indicates that the holiday maker can only depart on one of these dates, and he has only one departure date in the end. For instance, if 20 turned out to be true, then 6,13 and 27 would be false. In other words, the elements in the set can not coexist. The same applies to $[90,120]$. However, it is clear that the continuous grey number can not replace the discrete one in this case. If we replaced $\{6,13,20,27\}$ with $[6,27]$, then any number between them would be valid, such as 7 and 7.5 .

Obviously, grey numbers have great potential to complement other representations of information incompleteness. For example, the relational model requires a single value for each attribute value in its columns. A missing number is usually represented as null in a relational database [8]. It is represented as an interval [24] or a rough set [37] in non-deterministic information systems. However, both intervals and rough sets can be interpreted as multiple values and it can not differentiate multiple values from a single value represented by a set. A grey number appears to be a better choice under such a situation.

\section{Grey sets}

Similar to grey numbers, we could classify sets into three different categories:

Definition 16 (White sets). For a set $A \subseteq U$, if its characteristic function value of each $x$ with respect to $A$ can be expressed with a single white number $v \in[0,1]$

$$
\chi_{A}: U \rightarrow[0,1]
$$

then $A$ is a white set. 
In fact, type-1 fuzzy sets can be considered as a special case of white sets. A crisp set is clearly a white set and it is not fuzzy at all, but a type- 1 fuzzy set is still a white set although it is fuzzy compared with a crisp set.

Definition 17 (Black sets). For a set $A \subseteq U$, if its characteristic function value of each $x$ with respect to $A$ can be expressed with a black number, then $A$ is a black set.

An element in a black set has a complete unknown characteristic function value, and it is opposite to a white set where we have complete knowledge about the characteristic function value of an element. Between the two extremes, a set with incomplete information about its characteristic function values is defined as a grey set.

Definition 18 (Grey sets). For a set $A \subseteq U$, if the characteristic function value of $x$ with respect to $A$ can be expressed with a grey number $g_{A}^{ \pm}(x) \in$ $\bigcup_{i=1}^{n}\left[a_{i}^{-}, a_{i}^{+}\right] \in D[0,1]^{ \pm}$

$$
\chi_{A}: U \rightarrow D[0,1]^{ \pm}
$$

then $A$ is a grey set.

Here, $D[0,1]^{ \pm}$refers to the set of all grey numbers within the interval $[0,1]$. Similar to the expression of a fuzzy set, a grey set $A$ is represented with its relevant elements and their associated grey numbers for characteristic function:

$$
A=g_{A}^{ \pm}\left(x_{1}\right) / x_{1}+g_{A}^{ \pm}\left(x_{2}\right) / x_{2}+\ldots+g_{A}^{ \pm}\left(x_{n}\right) / x_{n}
$$

The characteristic function here is a general expression, it does not exclude any relevant criteria in defining a set. Therefore, it can be replaced by probability function, membership function, possibility function and etc. For a white set, we know clearly the relationship between an element and a set. Obviously, a white set here is different from a crisp set in traditional sets. A white set has a clear relationship between the set and relevant elements, and that relationship is not necessarily a crisp relationship. If we replaced the characteristic function with a 
fuzzy membership function, then the white set would become a standard type-1 fuzzy set.

Example 2. Following the previous example, we consider 4 people planning their holiday: a, b, c and d. Each person can visit 1 to 6 places in his holiday. Each person has petrol allowance of 120 litres for the holiday. The possible visits are:

- a: 1 or 2 places

- b: $2-4$ places

- c: $3-5$ places

- d: 6 places

To simplify our consideration, we assume that for each place the petrol consumption is between 15 and 20 litres. Consider two sets: $A$ is a set for the completeness of their visit to the 6 places, $\mathrm{B}$ is a set for the full consumption of their petrol. The characteristic functions of $A$ and $B$ are defined as

$$
f_{A}=\frac{n}{6} \quad f_{B}=\frac{l}{120}
$$

where $n$ is the number of places one person visited, and $l$ is the amount of petrol one person consumed during his holiday. Then $A$ and $B$ are two grey sets, their characteristic function values are:

- $f_{A}(a)=\left\{\frac{1}{6}, \frac{1}{3}\right\}, f_{B}(a)=\left[\frac{1}{8}, \frac{1}{3}\right]$

- $f_{A}(b)=\left\{\frac{1}{3}, \frac{1}{2}, \frac{2}{3}\right\}, f_{B}(b)=\left[\frac{1}{4}, \frac{2}{3}\right]$

- $f_{A}(c)=\left\{\frac{1}{2}, \frac{2}{3}, \frac{5}{6}\right\}, f_{B}(c)=\left[\frac{3}{8}, \frac{5}{6}\right]$

- $f_{A}(d)=1, f_{B}(d)=\left[\frac{3}{4}, 1\right]$

Obviously, the two sets $A$ and $B$ need different representation for their characteristic function values, and only the proposed grey sets could satisfy this requirement. 


\begin{tabular}{|c|c|c|c|}
\hline \hline Name & Gender & Working Attitude & Exam Result \\
\hline \hline Mike & Male & Good & Good \\
Jane & Female & Neutral & Good \\
Claire & Female & Neutral & Neutral \\
David & Male & Neutral & Poor \\
Lisa & Female & Poor & Poor \\
\hline \hline
\end{tabular}

Table 1: Information for 5 people

Example 3. There are five students in table 1, their name, gender, working altitude and exam results are listed in the table. A set $A$ for evaluating the study of students is to be established with respect to different attributes. Assume $a_{i}$ is a student in the table, and $i=1,2,3, \ldots, n$. $n$ is the number of students. We can get $A$ directly from Exam Result attribute in the table, and it is also possible to establish $A$ indirectly using other attributes, such as Working Attitude and Gender. We adopt Working Attitude to establish a grey set.

The Exam Result shows some kind of relationships with Working Attitude. A characteristic function is established according to the relationship between Working Attitude and Exam Result:

$$
f_{A}{ }^{c}\left(a_{i}\right)=\left\{\begin{array}{lr}
1 & \text { if } a_{i} \text { 's Working Attitude = good; } \\
{[0,1]} & \text { if } a_{i} \text { 's Working Attitude = neutral; } \\
0 & \text { if } a_{i} \text { 's Working Attitude = poor. }
\end{array}\right.
$$

Under this characteristic function, $A=[1,1] /$ Mike $+[0,1] /$ Jane $+[0,1] /$ Claire + $[0,1] /$ David $+[0,0] /$ Lisa $=1 /$ Mike $+[0,1] /$ Jane $+[0,1] /$ Claire $+[0,1] /$ David + $0 /$ Lisa. Obviously, $A$ is a grey set.

It is clear that a grey set has ill defined relationships between some elements and the set, and their characteristic functions have a grey number for a given attribute value.

From this example, it is clear that there are two groups of students in a grey set $A$ according to their characteristic function values: students with white numbers (0 or 1$)$ and students with grey numbers. They are two different categories. We can classify the elements relevant to a grey set into three different 
categories: white, grey and black elements.

Definition 19 (Category of elements in a set). Let $A$ be a grey set and $A \subseteq U$. For $x \in U, g_{A}^{ \pm}(x)$ is the value for characteristic function of $x$ with respect to $A$.

- If $g_{A}^{ \pm}(x)$ is a white number, then $x$ is called a white element

- If $g_{A}^{ \pm}(x)$ is a black number, then $x$ is called a black element

- If $g_{A}^{ \pm}(x)$ is a grey number, then $x$ is called a grey element

Because of the existence of grey and black elements, the relationships between some elements and a grey set are not completely known. The value for its corresponding characteristic function can only be expressed as a grey number. This is caused by the incomplete information of this element. Similar to the case for a grey number, the uncertainty caused by the information incompleteness can be measured using a degree of greyness. Considering the specific feature of grey sets, the degree of greyness for an element and a set are defined here.

Definition 20 (Degree of greyness for an element). Let $U$ be the finite universe of discourse, $x$ be an element and $x \in U$. For a grey set $A \subseteq U$, the characteristic function value of $x$ with respect to $A$ is $g_{A}^{ \pm}(x) \in D[0,1]^{ \pm}$. The degree of greyness $g_{A}^{\circ}(x)$ of element $x$ for set $A$ is expressed as

$$
g_{A}^{\circ}(x)=\left|g^{+}-g^{-}\right|
$$

Based on the degree of greyness for an element, a degree of greyness for a set is defined as follow.

Definition 21 (Degree of greyness for a set). Let $U$ be the finite universe of discourse, $A$ be a grey set and $A \subseteq U . x_{i}$ is a an element relevant to $A$ and $x_{i} \in U . i=1,2,3, \ldots, n$ and $n$ is the cardinality of $U$. The degree of greyness of set $A$ is defined as

$$
g_{A}^{\circ}=\frac{\sum_{i=1}^{n} g_{A}^{\circ}\left(x_{i}\right)}{n}
$$


According to the given definition, the uncertainty caused by incomplete information for the evaluation of students under different attributes can be measured using the degree of greyness for elements and sets.

Example 4. The degree of greyness for Jane, Claire and David in Example 3 could be calculated as

$$
\begin{gathered}
g^{\circ}(\text { Jane })=1-0=1 \\
g^{\circ}(\text { Mike })=1-1=0 \\
g^{\circ}(\text { Lisa })=0-0=0
\end{gathered}
$$

For the grey set $A$ derived from Working Attitude, its degree of greyness is

$$
g_{G}^{\circ}=\frac{0+1+1+1+0}{5}=0.6
$$

\begin{tabular}{|c|c|c|c|c|c|c|}
\hline \multirow[t]{2}{*}{ Name } & \multicolumn{2}{|c|}{ Exam Result } & \multicolumn{2}{|c|}{ Working Attitude } & \multicolumn{2}{|c|}{ Gender } \\
\hline & Element & Set & Element & Set & Element & Set \\
\hline Mike & 0 & \multirow{5}{*}{0} & 0 & \multirow{5}{*}{0.6} & 1 & \multirow{5}{*}{1} \\
\hline Jane & 0 & & 1 & & 1 & \\
\hline Claire & 0 & & 1 & & 1 & \\
\hline David & 0 & & 1 & & 1 & \\
\hline Lisa & 0 & & 0 & & 1 & \\
\hline
\end{tabular}

The results for the sets evaluated according to Exam Result, Working Attitude and Gender are shown in Table 2.

Table 2: Example for degree of greyness

From Table 2, it is clear that a white set has a degree of greyness of 0 , a black set has a degree of greyness of 1 and a grey set has a degree of greyness between 0 and 1 .

The following results relate grey sets, fuzzy sets, non-deterministic information systems and rough sets.

Theorem 2. Let $U$ be the finite universe of discourse, and $A$ be a grey set and $A \subseteq U . x$ is an element and $x \in U, g_{A}^{ \pm}(x)$ is the characteristic function value of $x$ with respect to $A, g_{A}^{\circ}(x)$ is the degree of greyness of $g_{A}^{ \pm}(x)$, and $g_{A}^{\circ}$ is the degree of greyness for $A$. The following properties hold for $x$ and $A$ : 
- $A$ is a white set iff $g_{A}^{\circ}=0$

- $A$ is a black set iff $g_{A}^{\circ}=1$

- $A$ is a crisp set iff $g_{A}^{\circ}=0$ and $g_{A}^{ \pm}(x) \in\{0,1\}$ for any $x \in U$

- $A$ is a type- 1 fuzzy set iff $g_{A}^{\circ}=0$ and $g_{A}^{ \pm}(x) \in[0,1]$ for any $x \in U$

- $A$ is a shadow set iff $0<g_{A}^{\circ}<1$ and $g_{A}^{ \pm}(x) \in\{0,1,[0,1]\}$ for any $x \in U$

- $A$ is an interval-valued fuzzy set iff $g_{A}^{ \pm}(x)$ is a continuous grey number for any $x \in U$ and an interval is interpreted as a representation of a single unknown value with known boundary

Proof These rules are clear and not difficult to prove. We provide a proof only for the the final property. If we consider the characteristic function as a fuzzy membership function $\mu$, then we have

$$
\mu_{A}: U \rightarrow D[0,1]^{ \pm}
$$

For a continuous grey number, $g^{ \pm} \in\left[g^{-}, g^{+}\right] \in D[0,1]^{ \pm}$can be considered as an interval $d$ representing an unknown value and $d \in D[0,1]$. Hence the grey set $A$ can be expressed as

$$
\mu_{A}: U \rightarrow D[0,1]
$$

this is an interval-valued fuzzy set. For an interval-valued fuzzy set, the fuzzy membership $\mu$ can be considered as characteristic function, then we have

$$
\chi_{:} U \rightarrow D[0,1]
$$

Assume the membership interval $d=\left[g^{-}, g^{+}\right]$, which is an interval representing an unknown value within boundary of $\left[g^{-}, g^{+}\right]$. Clearly, this interval is a continuous grey number $g^{ \pm} \in d$, then we have a grey set with $\mu$ as characteristic function.

$$
\mu: U \rightarrow D[0,1]^{ \pm}
$$


This theorem shows that grey sets extend crisp sets, fuzzy sets and intervalvalued fuzzy sets. According to theorem 1, a grey number could be in three different situations, and an interval is only one of these situations. When the corresponding grey numbers are represented by discrete sets or mixed sets, they are completely different from interval-valued fuzzy sets and cannot be replaced by interval-valued fuzzy sets. From Theorem 2, a grey set is only equivalent to an interval-valued fuzzy set when it has interval grey numbers as its characteristics function values and an interval in the interval-valued fuzzy sets is interpreted as a an unknown single value with a known boundary. Both conditions are essential for a grey set to be equivalent to an interval-valued fuzzy set. For example, a noisy flight represented with an interval-valued fuzzy set could be interpreted as perceptions of a group of observers and it is an inclusive aggregation: each membership value inside this interval is valid and they do not exclude each other. However, a noisy flight represented with a grey set refers only to individual's perception and its representation is exclusive: only one membership could be true. Therefore, a grey set may have semantic difference from an interval-valued fuzzy set even if both employ intervals as their representation. Another proof to this conclusion is their relationship with non-deterministic information systems.

Theorem 3. A fuzzified non-deterministic information system is a grey set.

Proof $A$ is a non-deterministic information system and $A=\left(O B, A T,\left\{V A L_{a} \mid a \in\right.\right.$ $A T\}, g)$. Now, we consider each attribute as a fuzzy set and fuzzify their attribute values into fuzzy membership values for their fuzzy attribute sets: $A_{g}=$ $\left(O B, A T_{g},\left\{V A L_{a_{g}} \mid a_{g} \in A T_{g}\right\}, g_{g}\right)$. Here, $A T_{g}$ is a finite set whose elements are fuzzy sets for attributes, $V A L_{a_{g}}$ is a finite set whose elements are membership values of corresponding fuzzy attribute sets, $g_{g}$ is a mapping from $O B \times A T_{g}$ to a power set of $\cup_{a_{g} \in A T_{g}} V A L_{a_{g}}$, i.e., $g_{g}: O B \times A T_{g} \rightarrow P\left(\cup_{a_{g} \in A T_{g}} V A L_{a_{g}}\right)$. It is clear that $g_{g}(x, a) \in P\left(\cup_{a_{g} \in A T_{g}} V A L_{a_{g}}\right)$ may be a finite set which is not representable by an interval. According to the definition of non-deterministic information systems, $g_{g}\left(x, a_{g}\right)$ is interpreted as if there is an actual value in this set but it is not known. Obviously, $g_{g}\left(x, a_{g}\right) \in D[0,1]^{ \pm}$is a grey number. If we 
consider $U=O B \times A T_{g}$ as the finite universe, then we have

$$
\chi_{A_{g}}: U \rightarrow D[0,1]^{ \pm}
$$

Therefore, $A_{g}$ is a grey set.

Theorem 3 demonstrates that a grey set combines the representation of incompleteness together with vagueness and is more powerful than an intervalvalued fuzzy set.

Theorem 4. $A$ is a rough set iff $g_{A}^{\circ}>0$ and $g_{A}^{ \pm}(x) \subseteq\{0,1\}$ holds for any $x \in U . g_{A}^{ \pm}(x)$ refers to the characteristic function value of $x$ with respect to $A \subseteq U . g_{A}^{\circ}$ is the degree of greyness of $A$.

Proof If $g_{A}^{ \pm}(x) \subseteq\{0,1\}$ holds for any $x \in U$, then $g_{A}^{ \pm}(x)$ is a discrete grey number, and $g_{A}^{ \pm}(x) \in\{0,1\}$. There are only three options for the result value of $g_{A}^{ \pm}(x): 0,1$ or $\{0,1\}$. The elements in $U$ can be classified into three different crisp sets according to the value of $g_{A}^{ \pm}(x): \underline{A}$ for $g_{A}^{ \pm}(x)=1, F$ for $g_{A}^{ \pm}(x)=\{0,1\}$ and $\sim A$ for $g_{A}^{ \pm}(x)=0$. Obviously, $\underline{A} \cap \sim A=\emptyset$. If $g_{A}^{\circ}>0$, then $F \neq \emptyset$. The elements in $F$ are not determined, and they may belong to $\underline{A}$ or $\sim A$ with more information. There are two possible extreme situations: $g_{A}^{ \pm}(x)=1$ for each $x \in F$ or $g_{A}^{ \pm}(x)=0$ for each $x \in F$. For the first situation, we get the maximum $\bar{A}=\underline{A} \cup F$. For the second situation, we get the minimum $\underline{A}$. Obviously, $\bar{A} \supseteq A \supseteq \underline{A}$. Let $R=U \times U$ be an equivalence relation on the universe $U$, and $[x]_{R}$ be the equivalence class containing $x$. Thus each $x \in U$ represents an equivalent class $[x]_{R}$. The elements in $[x]_{R}$ should include all validate characteristic function values for $x \in U$. From our analysis of $\underline{A}$ and $\bar{A}$, we have

- $g_{A}^{ \pm}\left(x_{i}\right)=1$ if $x_{i} \in[x]_{R}$ and $[x]_{R} \subseteq \underline{A}$

- There is at least one element $x_{i}$ satisfying $g_{A}^{ \pm}\left(x_{i}\right)=1$ for each $x_{i} \in[x]_{R}$ if $[x]_{R} \subseteq \bar{A}$

$i$ is the index of the elements in $[x]_{R}, i=1,2, \ldots, k$. $k$ is the number of the elements in $[x]_{R}$. Therefore, we have 
- $[x]_{R} \subseteq A$ iff $x \in \underline{A}$

- $[x]_{R} \cap A \neq \emptyset$ if $x \in \bar{A}$

Then we have the lower approximation $\underline{a p r}_{R}(A)$ and the upper approximation $\overline{a p r}_{R}(A)$ as follows:

- $\underline{a p r}_{R}(A)=\left\{x \in U \mid[x]_{R} \subseteq A\right\}=\underline{A}$

- $\overline{a p r}_{R}(A)=\left\{x \in U \mid[x]_{R} \cap A \neq \Phi\right\}=\bar{A}$

Obviously, under the given condition, a grey set is equivalent to a rough set. For a rough set $A$, it satisfies the two equations above. A characteristic function could be established:

$$
f_{A}{ }^{c}(x)=\left\{\begin{array}{lr}
1 & \text { if } x \in \underline{a p r}_{R}(A) ; \\
\{0,1\} & \text { if } x \in \overline{a p r}_{R}(A) \text { but } x \notin \frac{a p r}{R}(A) ; \\
0 & \text { if } x \notin \overline{a p r}_{R}(A) .
\end{array}\right.
$$

Obviously, the value of this characteristic function contains discrete grey number. Then $A$ satisfies

$$
\chi_{A}: U \rightarrow D[0,1]^{ \pm}
$$

This is a grey set.

Similar to fuzzy sets, an $\alpha$ cut of a grey set $A$ is defined as:

$$
A_{\alpha}=\left\{x \in A \mid g_{A}^{ \pm}(x) \geq \alpha\right\}
$$

Obviously, the following corollary about $\alpha$ cuts can be draw from Theorem 4:

Corollary 1. The $\alpha$ cut of a grey set is a rough set.

Theorem 4 proves that grey sets include rough sets as a special case in the case of a finite universe of discourse. Therefore, a rough set is a special grey set. Actually, we have the following theorem for the link between roughness and greyness. 
Theorem 5. $A$ is a grey set where $g_{A}^{\circ}>0, g_{A}^{ \pm}(x) \subseteq\{0,1\}$ holds for any $x \in U$, and $(\underline{A}, \bar{A})$ is the equivalent rough set representation of $A . g_{A}^{\circ}$ is the degree of greyness of $A$, and $R^{\circ}(A)$ is the roughness of $A$. Then we have

$$
g_{A}^{\circ}=\frac{R^{\circ}(A) R^{\circ}(\neg A)}{R^{\circ}(A)+R^{\circ}(\neg A)-R^{\circ}(A) R^{\circ}(\neg A)}
$$

Proof From theorem 4, we know that the grey representation and rough representation of $\mathrm{A}$ are equivalent to each other, and

$$
\chi_{A}(x)=\left\{\begin{array}{lr}
1 & \text { if } x \in \underline{A} ; \\
\{0,1\} & \text { if } x \in \bar{A} \text { but } x \notin \underline{A} ; \\
0 & \text { if } x \notin \bar{A} .
\end{array}\right.
$$

Thus, we have

$$
g_{A}^{\circ}(x)=\left\{\begin{array}{rr}
0 & \text { if } x \in \underline{A} ; \\
1 & \text { if } x \in \bar{A} \text { but } x \notin \underline{A} ; \\
0 & \text { if } x \notin \bar{A} .
\end{array}\right.
$$

From Definition 7, we have

$$
g_{A}^{\circ}=\frac{\sum g_{A}^{\circ}(x)}{n}=\frac{1}{\frac{|\bar{A}|}{|\bar{A}-\underline{A}|}+\frac{|\neg A|+|\bar{A}-\underline{A}|}{|\bar{A}-\underline{A}|}-1}
$$

However

$$
R^{\circ}(A)=\frac{|\bar{A}-\underline{A}|}{|\bar{A}|} \quad \text { and } \quad R^{\circ}(\neg A)=\frac{|\bar{A}-\underline{A}|}{|\bar{A}|+|\bar{A}-\underline{A}|}
$$

Hence, we have

$$
g_{A}^{\circ}=\frac{R^{\circ}(A) R^{\circ}(\neg A)}{R^{\circ}(A)+R^{\circ}(\neg A)-R^{\circ}(A) R^{\circ}(\neg A)}
$$

Theorem 5 indicates that the degree of greyness has close relationship with the roughness of a set, and the proposed degree of greyness of a set integrates roughness together with fuzziness.

Rough sets actually provide a method to construct interval sets, hence the conclusions in Theorem 4 and Corollary 1 hold for interval sets as well. In our recent work on R-Fuzzy sets, we have proved that a grey set is a special case of R-fuzzy sets [44]. 


\section{Grey subsets and their operations}

Clearly, grey sets have close relationships with interval-valued fuzzy sets and intuitionistic fuzzy sets. However, because of the existence of the whitenisation function, their operations are not exactly the same. First, we introduce the concept of whitenisation of a grey set and consistent whitenisation functions.

Definition 22 (Whitenisation of grey sets). $A$ is a grey set and $A \subseteq U . x$ is an element and $x \in U . g_{A}^{\circ}(x)$ is the degree of greyness of $x$ with respect to $A$. $g_{A}^{-}(x)$ and $g_{A}^{+}(x)$ are the lower and upper limits of $g_{A}^{ \pm}(x) . \nabla g_{A}^{ \pm}(x)=F\left(g_{A}^{ \pm}(x)\right)$ is a whitenisation function of $g_{A}^{ \pm}(x)$. A whitenisation of $A$ is a new grey set $\nabla A$

$$
\nabla A=\left\{\left\langle x, \nabla g_{A}^{ \pm}(x)\right\rangle: x \in U\right\}
$$

Similar to the whitenisation of grey numbers, the whitenisation of grey sets is in fact a process to remove the uncertainty caused by incomplete information. Obviously, we have

$$
g_{\nabla A}^{\circ}<g_{A}^{\circ}
$$

As a result of whitenisation, we get a whiter grey set, and we get a white set when the whitenisation function is a complete whitenisation function.

Definition 23 (Consistent whitenisation). $A \subseteq U$ and $B \subseteq U$ are two grey sets, $\nabla A$ and $\nabla B$ are their complete whitenisations. $x \in U$ is an element. $g_{A}^{ \pm}(x), g_{B}^{ \pm}(x), g_{\nabla A}(x)$ and $g_{\nabla B}(x)$ are values of the characteristic functions of $x$ with respect to $A, B, \nabla A$ and $\nabla B . g_{A}^{-}(x), g_{A}^{+}(x), g_{B}^{-}(x)$ and $g_{B}^{+}(x)$ are the lower and upper limits of $g_{A}^{ \pm}(x)$ and $g_{B}^{ \pm}(x)$. We call $A$ consistent with $B$ if the following conditions hold for any $x \in U$ :

$$
g_{\nabla A}(x) \leq g_{\nabla B} \text { if } g_{A}^{-}(x) \leq g_{B}^{-}(x) \text { and } g_{A}^{+}(x) \leq g_{B}^{+}(x)
$$

In grey systems, each grey number is associated with a complete whitenisation function. This function is usually unknown. However, it can be completely different from one grey number to another. That means the underlying white 
numbers may not be the same even if their upper and lower limits are exact the same. Therefore, the subset of a grey set has additional requirement than those in interval-valued or intuitionistic fuzzy sets.

Definition 24 (Grey subsets). $A$ and $B$ are two grey sets, $A \subseteq U$ and $B \subseteq$ $U . x \in U$ is an element. $g_{A}^{ \pm}(x)$ and $g_{B}^{ \pm}(x)$ are the characteristic function values of $x$ for $A$ and $B . g_{A}^{-}(x), g_{A}^{+}(x), g_{B}^{-}(x)$ and $g_{B}^{+}(x)$ are the upper and lower limits of $g_{A}^{ \pm}(x)$ and $g_{B}^{ \pm}(x) . A$ is a subset of $B$ if the following conditions hold

- For any $x \in U$, we have $g_{A}^{-}(x) \leq g_{B}^{-}(x)$ and $g_{A}^{+}(x) \leq g_{B}^{+}(x)$

- $A$ is consistent with $B$

It is represented as $A \subseteq B$.

With this definition of subset, we have

$$
A=B \Leftrightarrow A \subseteq B \text { and } B \subseteq A
$$

Example 5. In Example 2, we have two grey sets $A$ and $B$ :

$$
\begin{gathered}
A=\left\{\frac{1}{6}, \frac{1}{3}\right\} / a+\left\{\frac{1}{3}, \frac{1}{2}, \frac{2}{3}\right\} / b+\left\{\frac{1}{2}, \frac{2}{3}, \frac{5}{6}\right\} / c+1 / d \\
B=\left[\frac{1}{8}, \frac{1}{3}\right] / a+\left[\frac{1}{4}, \frac{2}{3}\right] / b+\left[\frac{3}{8}, \frac{5}{6}\right] / c+\left[\frac{3}{4}, 1\right] / d
\end{gathered}
$$

The degree of greyness for each element of $A$ and $B$ :

$$
\begin{gathered}
g_{A}^{\circ}(a)=\frac{1}{6}, \quad g_{A}^{\circ}(b)=\frac{1}{3}, \quad g_{A}^{\circ}(c)=\frac{1}{3} \quad \text { and } \quad g_{A}^{\circ}(d)=0 \\
g_{B}^{\circ}(a)=\frac{5}{24}, \quad g_{B}^{\circ}(b)=\frac{5}{12}, \quad g_{B}^{\circ}(c)=\frac{11}{24} \quad \text { and } \quad g_{B}^{\circ}(d)=\frac{1}{3}
\end{gathered}
$$

The degree of greyness of $A$ and $B$ :

$$
\begin{gathered}
g_{A}^{\circ}=\frac{\frac{1}{6}+\frac{1}{3}+\frac{1}{3}+0}{4}=\frac{5}{24} \\
g_{B}^{\circ}=\frac{\frac{5}{24}+\frac{5}{12}+\frac{11}{24}+\frac{1}{4}}{4}=\frac{1}{3}
\end{gathered}
$$


For $A$, let $\nabla g_{A}^{ \pm}(x)=g_{A}^{ \pm}(x)-\left\{g_{A}^{-}(x)\right\}$ if $g_{A}^{\circ}(x)>0$ and $\nabla g_{A}^{ \pm}(x)=g_{A}^{+}(x)$ if $g_{A}^{\circ}(x)=0$, we get a whiter set $\nabla A$ :

$$
\nabla A=\frac{1}{3} / a+\left\{\frac{1}{2}, \frac{2}{3}\right\} / b+\left\{\frac{2}{3}, \frac{5}{6}\right\} / c+1 / d
$$

For $B$, let $\nabla g_{B}^{ \pm}(x)=g_{B}^{ \pm}(x)-\left[g_{B}^{+}(x)-\frac{g_{B}^{+}(x)-g_{B}^{-}(x)}{10}, g_{B}^{+}(x)\right]$, we have a whiter set $\nabla B:$

$$
\nabla B=\left[\frac{1}{8}, \frac{5}{16}\right] / a+\left[\frac{1}{4}, \frac{5}{8}\right] / b+\left[\frac{3}{8}, \frac{63}{80}\right] / c+\left[\frac{3}{4}, \frac{29}{30}\right] / d
$$

The degree of greyness for each element of $\nabla A$ and $\nabla B$ :

$$
\begin{gathered}
g_{\nabla A}^{\circ}(a)=0, \quad g_{\nabla A}^{\circ}(b)=\frac{1}{6}, \quad g_{\nabla A}^{\circ}(c)=\frac{1}{6} \quad \text { and } \quad g_{\nabla A}^{\circ}(d)=0 \\
g_{\nabla B}^{\circ}(a)=\frac{3}{16}, \quad g_{\nabla B}^{\circ}(b)=\frac{3}{8}, \quad g_{\nabla B}^{\circ}(c)=\frac{33}{80} \text { and } \quad g_{\nabla B}^{\circ}(d)=\frac{13}{60}
\end{gathered}
$$

The degree of greyness of $\nabla A$ and $\nabla B$ :

$$
\begin{gathered}
g_{\nabla A}^{\circ}=\frac{0+\frac{1}{6}+\frac{1}{6}+0}{4}=\frac{1}{12} \\
g_{\nabla B}^{\circ}=\frac{\frac{3}{16}+\frac{3}{8}+\frac{33}{80}+\frac{13}{60}}{4}=\frac{3}{10}
\end{gathered}
$$

For each $x \in U=\{a, b, c, d\}$, we have

$$
g_{\nabla A}^{\circ}(x)<g_{A}^{\circ}(x) \text { and } g_{\nabla B}^{\circ}(x)<g_{B}^{\circ}(x)
$$

For the degree of greyness of $A, \nabla A, B$ and $\nabla B$, we have

$$
g_{\nabla A}^{\circ}<g_{A}^{\circ} \text { and } g_{\nabla B}^{\circ}<g_{B}^{\circ}
$$

Obviously, $\nabla A$ is a whitenisation of $A$ and $\nabla B$ is a whitenisation of $B$.

For any $x \in U=\{a, b, c, d\}$, if we assume $g_{\nabla A}^{ \pm}(x)=g_{A}^{+}(x)$ and $g_{\nabla B}^{ \pm}(x)=$ $\frac{g_{B}^{+}(x)+g_{B}^{-}(x)}{2}$, we have

$$
g_{\nabla B}^{ \pm}(x) \leq g_{\nabla A}^{ \pm}, \quad g_{B}^{-}(x) \leq g_{A}^{-}(x) \quad \text { and } \quad g_{B}^{+}(x) \leq g_{A}^{+}(x)
$$

Clearly, $B$ is consistent with $A$ under the given assumptions, and we have

$$
B \subseteq A
$$


As a set, grey set has similar set operations for union, intersection and complement. $A \subseteq U$ and $B \subseteq U$ are two grey sets, and $x \in U$ is an element. $g_{A}^{ \pm}(x) \in \bigcup_{i=1}^{m}\left[a_{i}^{-}, a_{i}^{+}\right]$and $g_{B}^{ \pm}(x) \in \bigcup_{i=1}^{n}\left[b_{i}^{-}, b_{i}^{+}\right]$are the characteristic function values of $x$ for $A$ and $B . g_{A}^{-}(x), g_{A}^{+}(x), g_{B}^{-}(x)$ and $g_{B}^{+}(x)$ are the upper and lower limits of $g_{A}^{ \pm}(x)$ and $g_{B}^{ \pm}(x)$.

Definition 25 (Union of grey sets). The union of $A$ and $B$ is a new grey set $C$

$$
C=A \cup B=\left\{\left\langle x, g_{C}^{ \pm}(x)\right\rangle: x \in U\right\}
$$

where $g_{C}^{ \pm}(x)$ is the characteristic function value of $x$ for $C$, its upper and lower limits are $g_{C}^{-}(x)$ and $g_{C}^{+}(x)$

$$
g_{C}^{ \pm}(x)=\bigcup_{i=1}^{m}\left[a_{i}^{-}, a_{i}^{+}\right] \cup \bigcup_{i=1}^{n}\left[b_{i}^{-}, b_{i}^{+}\right]-\left[g_{A}^{-}(x) \wedge g_{B}^{-}(x), g_{A}^{-}(x) \vee g_{B}^{-}(x)\right)
$$

Theorem 6. The degree of greyness $g_{C}^{\circ}(x)$ of $x$ for grey set $C=A \cup B$ satisfies

$$
g_{A}^{\circ}(x) \wedge g_{B}^{\circ}(x) \leq g_{C}^{\circ}(x) \leq g_{A}^{\circ}(x) \vee g_{B}^{\circ}(x)
$$

Proof Assume the value of characteristic function for $x$ in $A, B$ and $C$ are $g_{A}^{ \pm}(x), g_{B}^{ \pm}(x)$ and $g_{C}^{ \pm}(x)$. According to Definition 20, we have

$$
\begin{aligned}
& g_{A}^{\circ}(x)=g_{A}^{+}(x)-g_{A}^{-}(x) \\
& g_{B}^{\circ}(x)=g_{B}^{+}(x)-g_{B}^{-}(x) \\
& g_{C}^{\circ}(x)=g_{C}^{+}(x)-g_{C}^{-}(x)
\end{aligned}
$$

From Definition 25, we have

$$
g_{C}^{\circ}(x)=g_{A}^{+}(x) \vee g_{B}^{+}(x)-g_{A}^{-}(x) \vee g_{B}^{-}(x)
$$

Assume $g_{A}^{+}(x) \geq g_{B}^{+}(x)$, then we have two different situations:

- $g_{A}^{-}(x) \geq g_{B}^{-}(x)$, we have

$$
g_{C}^{\circ}(x)=g_{A}^{+}(x)-g_{A}^{-}(x)=g_{A}^{\circ}(x)
$$


- $g_{A}^{-}(x)<g_{B}^{-}(x)$, we have

$$
g_{C}^{\circ}(x)=g_{A}^{+}(x)-g_{B}^{-}(x)<g_{A}^{+}(x)-g_{A}^{-}(x)=g_{A}^{\circ}(x)
$$

and

$$
g_{C}^{\circ}(x)=g_{A}^{+}(x)-g_{B}^{-}(x)>g_{B}^{+}(x)-g_{B}^{-}(x)=g_{B}^{\circ}(x)
$$

Similarly, if $g_{A}^{+}(x)<g_{B}^{+}(x)$, we have

- $g_{A}^{-}(x) \leq g_{B}^{-}(x)$, we have

$$
g_{C}^{\circ}(x)=g_{B}^{+}(x)-g_{B}^{-}(x)=g_{B}^{\circ}(x)
$$

- $g_{A}^{-}(x)>g_{B}^{-}(x)$, we have

$$
g_{C}^{\circ}(x)=g_{B}^{+}(x)-g_{A}^{-}(x)>g_{A}^{+}(x)-g_{A}^{-}(x)=g_{A}^{\circ}(x)
$$

and

$$
g_{C}^{\circ}(x)=g_{B}^{+}(x)-g_{A}^{-}(x)<g_{B}^{+}(x)-g_{B}^{-}(x)=g_{B}^{\circ}(x)
$$

Obviously, we have

$$
g_{A}^{\circ}(x) \wedge g_{B}^{\circ}(x) \leq g_{C}^{\circ}(x) \leq g_{A}^{\circ}(x) \vee g_{B}^{\circ}(x)
$$

Definition 26 (Intersection of grey sets). The intersection of $A$ and $B$ is a new grey set $C$

$$
C=A \cap B=\left\{\left\langle x, g_{C}^{ \pm}(x)\right\rangle: x \in U\right\}
$$

where $g_{C}^{ \pm}(x)$ is the characteristic function value of $x$ for $C$, its upper and lower limits are $g_{C}^{-}(x)$ and $g_{C}^{+}(x)$

$$
g_{C}^{ \pm}(x)=\bigcup_{i=1}^{m}\left[a_{i}^{-}, a_{i}^{+}\right] \cup \bigcup_{i=1}^{n}\left[b_{i}^{-}, b_{i}^{+}\right]-\left(g_{A}^{+}(x) \wedge g_{B}^{+}(x), g_{A}^{+}(x) \vee g_{B}^{+}(x)\right)
$$

Similar to union, there is a theorem for the degree of greyness of elements in the intersection of grey sets.

Theorem 7. The degree of greyness $g_{C}^{\circ}(x)$ of $x$ for grey set $C=A \cap B$ satisfies

$$
g_{A}^{\circ}(x) \wedge g_{B}^{\circ}(x) \leq g_{C}^{\circ}(x) \leq g_{A}^{\circ}(x) \vee g_{B}^{\circ}(x)
$$


This theorem can be proved in a similar way like union. We do not repeat it here.

Definition 27 (Complement of grey sets). The complement of $A$ is a new grey set $\sim A$

$$
\sim A=\left\{\left\langle x, g_{\sim A}^{ \pm}(x)\right\rangle: x \in U\right\}
$$

where $g_{\sim A}^{ \pm}(x)$ is the characteristic function value of $x$ for $\sim A$, its upper and lower limits are $g_{\sim A}^{-}(x)$ and $g_{\sim A}^{+}(x)$

$$
g_{\sim A}^{ \pm}(x)=\bigcup_{i=1}^{m}\left[1-a_{i}^{+}, 1-a_{i}^{-}\right]
$$

Theorem 8. The degree of greyness $g_{\sim A}^{\circ}(x)$ of $x$ for grey set $\sim A$ satisfies

$$
g_{\sim A}^{\circ}(x)=g_{A}^{\circ}(x)
$$

Similar to fuzzy sets, grey sets are concerning all elements in its domain, hence both $A$ and $\sim A$ are concerning the same domain $U$. They have the same cardinality without $\alpha$ cut. Therefore, we have the following corollary from theorem 8 .

Corollary 2. The degree of greyness of a complement grey set $\sim A$ is exactly the same as the degree of greyness of the grey set $A$

$$
g_{\sim A}^{\circ}=g_{A}^{\circ}
$$

In addition to union, intersection and complement operations, we define some new operations for grey sets: lower approximation and upper approximation.

Definition 28 (Lower and upper approximation of a grey set). $A$ is a grey set and $A \subseteq U . \quad x$ is an element and $x \in U . g_{A}^{ \pm}(x)$ is the value of characteristic function of $x$ with respect to $A . g_{A}^{-}(x)$ and $g_{A}^{+}(x)$ are the lower and upper limits of $g_{A}^{ \pm}(x)$. The lower approximation $\underline{A}$ and upper approximation $\bar{A}$ are two new sets

$$
\begin{aligned}
& \underline{A}=\left\{\left\langle x, g_{A}^{-}(x)\right\rangle: x \in U\right\} \\
& \bar{A}=\left\{\left\langle x, g_{A}^{+}(x)\right\rangle: x \in U\right\}
\end{aligned}
$$


For the lower and upper approximation of a grey set $A$, we have the following theorem.

Theorem 9. The relationship between a grey set $A$ and its lower and upper approximation $\underline{A}$ and $\bar{A}$ is

$$
\underline{A} \subseteq A \subseteq \bar{A}
$$

The lower and upper approximations are in fact special cases for whitenisation operation.

Theorem 10. The following relationships hold for the lower and upper approximations and whitenisation operation of a grey set

- $\underline{A}=\left.\nabla A\right|_{\nabla g_{A}^{ \pm}(x)=g_{A}^{-}(x)}$

- $\bar{A}=\left.\nabla A\right|_{\nabla g_{A}^{ \pm}(x)=g_{A}^{+}(x)}$

where $\nabla g_{A}^{ \pm}(x)=F(x)$ is a unified complete whitenisation function for all $x \in U$.

Proof According to Definition 22, we have

$$
\nabla A=\left\{\left\langle x, \nabla g_{A}^{ \pm}(x)\right\rangle: x \in U\right\}
$$

However, we know that $\nabla g_{A}^{ \pm}(x)=g_{A}^{-}(x)$ for each $x \in \underline{A}$ and $\nabla g_{A}^{ \pm}(x)=g_{A}^{+}(x)$ for each $x \in \bar{A}$, then

$$
\begin{aligned}
& \left.\nabla A\right|_{\nabla g_{A}^{ \pm}(x)=g_{A}^{-}(x)}=\left\{\left\langle x, g_{A}^{-}(x)\right\rangle: x \in U\right\} \\
& \left.\nabla A\right|_{\nabla g_{A}^{ \pm}(x)=g_{A}^{+}(x)}=\left\{\left\langle x, g_{A}^{+}(x)\right\rangle: x \in U\right\}
\end{aligned}
$$

From Definition 28, we have

$$
\begin{aligned}
& \underline{A}=\left\{\left\langle x, g_{A}^{-}(x)\right\rangle: x \in U\right\} \\
& \bar{A}=\left\{\left\langle x, g_{A}^{+}(x)\right\rangle: x \in U\right\}
\end{aligned}
$$

Obviously

$$
\begin{aligned}
& \underline{A}=\left.\nabla A\right|_{\nabla g_{A}^{ \pm}(x)=g_{A}^{-}(x)} \\
& \bar{A}=\left.\nabla A\right|_{\nabla g_{A}^{ \pm}(x)=g_{A}^{+}(x)}
\end{aligned}
$$




\section{Conclusions}

Fuzzy sets, rough sets and grey systems provide three different but overlapping models for the representation of uncertainties in sets. There has been considerable research in fuzziness, roughness and the combination of fuzzy sets and rough sets. However, greyness of sets and the difference between grey systems and interval-valued fuzzy sets are still not well investigated so far. Here, we define grey sets using grey numbers considering all possible situations rather than the interval representation only. Our results show that a grey set combines vagueness and incompleteness into one model and can be specified to intervalvalued fuzzy sets or rough sets under special situations. However, a grey set can represent situations not covered by interval-valued fuzzy sets or rough sets. Based on the notion of grey sets, the degree of greyness of a grey set and various set operations between grey sets are discussed.

\section{References}

[1] G. Alefeld and J. Herzberger. Introduction to Interval Computations. Academic Press Inc., New York, USA, 1983. Transl. by J. Rokne from the original German 'Einführung In Die Intervallrechnung'.

[2] K. Antanassov. Intuitionistic fuzzy sets. Physica-Verlag, Heidelberg - New York, 1999.

[3] M. Banerjee and M. K. Chakraborty. Algebras from rough sets. In S. Pal, L. Polkowski, and A. Skowron, editors, Rough-Neuro Computing: Techniques for Computing with Words, pages 157-184. Springer-Verlag, 2004.

[4] G. Beliakova, H. Bustinceb, D. Goswamic, U. Mukherjeed, and N. Pale. On averaging operators for atanassov's intuitionistic fuzzy sets. Information Sciences, 181(6):1116-1124, 2011.

[5] P. Burillo and H. Bustince. Entropy on intuitionistic fuzzy sets and on interval-valued fuzzy sets. Fuzzy Sets and Systems, 78:305-316, 1996. 
[6] H. Bustince and P. Burillo. Vague sets are intuitionistic fuzzy sets. Fuzzy Sets and Systems, 79:403-405, 1996.

[7] F. J. Cheng, S. H. Hui, and Y. C. Chen. Reservoir operation using grey fuzzy stochastic dynamic programming. Hydrological Processes, 16:23952408, 2002.

[8] E. Codd. A relational model of data for large shared data banks. Communication of the ACM, 13:377-387, 1970.

[9] C. Cornelis, K. T. Atanassov, and E. E. Kerre. Intuitionistic fuzzy sets and interval-valued fuzzy sets: a critical comparison. In M. Wagenknecht and R. Hampel, editors, Third EUSFLAT proceedings, pages 159-163, Zittau, Germany, September 2003. European Society for Fuzzy Logic and Technology.

[10] S. Coupland and R. John. New geometric inference techniques for type-2 fuzzy sets. International Journal of Approximate Reasoning, 49(1):198-211, 2008.

[11] V. Cutello and J. Montero. Non deterministic fuzzy classification systems. In Proceedings of the 6th International IEEE Conference on Fuzzy Systems, pages 1689-1694, Barcelona, Spain, 1997.

[12] S. P. Demri and E. S. Orlowska. Incomplete Information: Structure, Inference, Complexity. Springer-Verlag, 2002.

[13] J. Deng. The control problems of grey systems. Systems and Control Letters, 1982.

[14] J. Deng. Introduction to grey system theory. Journal of Grey Systems, 1(1):1-24, 1989.

[15] G. Deschrijver and E. Kerre. On the relationship between some extensions of fuzzy set theory. Fuzzy Sets and Systems, 133(2):227-235, 2003. 
[16] G. Deschrijver and E. Kerre. On the position of intuitionistic fuzzy set theory in the framework of theories modelling imprecision. Information Sciences, 177(8):1860-1866, 2007.

[17] D. Dubois, W. Ostasiewicz, and H. Prade. Fuzzy set: history and basic notions. In D. Dubois and H. Prade, editors, Fundamentals of fuzzy sets, pages 21-124. Kluwer, 2000.

[18] D. Dubois and H. Prade. Rough sets and fuzzy rough sets. International Journal of General Systems, 17:191-209, 1990.

[19] D. Dubois and H. Prade. The three semantics of fuzzy sets. Fuzzy Sets and Systems, 90(2):141-150, 1997.

[20] T. B. Iwinski. Algebraic approach to rough sets. Bulletin of the Polish Academy of Science and Mathematics, 35:673-683, 1987.

[21] G. J. Klir and B. Yuan. Fuzzy sets and fuzzy logic theory and applications. Prentice Hall P T R, Upper Saddle River - New Jersey, 2002.

[22] J. Komorowski, J. Z. Pawlak, L. Polkowski, and A. Skowron. Rough sets: a tutorial. In S. K. Pal and A. Skowron, editors, Rough fuzzy hybridization: a new trend in decision-making. Springer, Singapore, 1999.

[23] Y. Lin, M. Chen, and S. Liu. Theory of grey systems: Capturing uncertainties of grey information. kybernetics: The International Journal of Systems and Cybernetics, 33:196-218, 2004.

[24] W. Lipski. On databases with incomplete information. Journal of the ACM, 28:41-70, 1981.

[25] S. Liu, T. Gao, and Y. Dang. Grey systems theory and its applications. The Science Press of China, Beijing, 2000.

[26] S. Liu and Y. Lin. Grey Information Theory and Practical Applications. Springer, 2006. 
[27] J. Mendel and R. John. Type-2 fuzzy sets made simple. IEEE Transactions on Fuzzy Systems, 10(2):117-127, 2002.

[28] A. Mousavi and P. Jabedar-Maralani. Double-faced rough sets and rough communication. Information Sciences, 148:41-53, 2002.

[29] E. Orlowska and Z. Pawlak. Representation of nondeterministic information. Theoretical Computer Science, 29:27-39, 1984.

[30] Z. Pawlak. Rough sets. International Journal of Computer and Information Sciences, 11(5):341-356, 1982.

[31] Z. Pawlak. Information Systems. Theoretical Foundations. Wydawnictwa Naukowo-Techniczne, 1983.

[32] Z. Pawlak. Rough sets: theoretical aspects of reasoning about data. Kluwer Academic Publishers, Dordrecht, 1991.

[33] Z. Pawlak and A. Skowron. Rough membership functions. In R. Yager, M. Fedrizzi, and J. Kacprzyk, editors, Advances in the Dempster-Shafer Theory of Evidence, pages 251-271. John Wiley and Sons, 1994.

[34] Z. Pawlak, S. Wong, and W. Ziarko. Rough sets: probabilistic versus deterministic approach. International Journal of Man-Machine Studies, 29:81-95, 1988.

[35] W. Pedrycz. Shadowed sets: representing and processing fuzzy sets. IEEE Transactions on Systems, Man, and Cybernetics - Part B: Cybernetics, 28:103-109, 1998.

[36] S. Rasouli and B. Davvaz. Roughness in mv-algebras. Information Sciences, 180(5):737-747, 2010.

[37] H. Sakai. On a method to extract rules from a table with non-deterministic information: a rough sets based approach. Bulletin of Informatics and Cybernetics, 34(1):13-28, 2002. 
[38] R. Sambuc. Fonctions $\Phi$-floues. Application I'Aide au Diagnostic en Patholologie Thyroidienne. PhD thesis, Univ. Marseille, France, 1975.

[39] G. Wang and Y. He. Intuitionistic fuzzy sets and l-fuzzy sets. Fuzzy Sets and Systems, 110:271-274, 2000.

[40] Q. Wu and Z. Liu. Real formal concept analysis based on grey-rough set theory. Knowledge-Based Systems, 22:38-45, 2009.

[41] U. Wybraniec-Skardowska. On generalization of approximation space. Bulletin of the Polish Academy of Science and Mathematics, 37:51-61, 1989.

[42] D. Yamaguchi, G.-D. Li, and M. Nagai. A grey-based rough approximation model for interval data processing. Information Sciences, 177:4727-4744, 2007.

[43] Y. Yang. Extended grey numbers and their operations. In Proceedings of 2007 IEEE International Conference on Systems, Man and Cybernetics, pages 2181-2186, Montreal, Canada, October 2007.

[44] Y. Yang and C. Hinde. A new extension of fuzzy sets using rough sets: R-fuzzy sets. Information Sciences, 180(3):354-365, 2010.

[45] Y. Yang and R. John. Grey systems and interval valued fuzzy sets. In M. Wagenknecht and R. Hampel, editors, Third EUSFLAT proceedings, pages 193-197, Zittau, Germany, September 2003. European Society for Fuzzy Logic and Technology.

[46] Y. Yang and R. John. Roughness bounds in rough set operations. Information Sciences, 176:3256-3267, 2006.

[47] Y. Yang and R. John. Roughness bounds in set-oriented rough set operations. In Proc. FUZZ-IEEE 2006, pages 1461-1468, 2006.

[48] Y. Yang, R. John, and F. Chiclana. Grey sets, fuzzy sets and rough sets. In In Proceedings of the 5th International Conference on Recent Advances 
in Soft Computing, RASC 2004, Nottingham, UK, 16-18 December 2004, pages 348-353, 2004. (ISBN 1-84233-110-8).

[49] Y. Yang and S. Liu. Reliability of operations of grey numbers using kernels. Grey Systems: Theory and Application, 1(1):57-71, 2011.

[50] Y. Y. Yao. Interval-set algebra for qualitative knowledge representation. In Proceedings of the 5th IEEE International Conference on Cognitive Informatics, pages 370-374, 1993.

[51] Y. Y. Yao. Two views of the theory of rough sets in finite universe. International Journal of Approximate Reasoning, 15(4):291-317, 1996.

[52] Y. Y. Yao. A comparative study of fuzzy sets and rough sets. Information Sciences, 109(1-4):227-242, 1998.

[53] Y. Y. Yao. Constructive and algebraic methods of the theory of rough sets. Information Sciences, 109(1-4):21-47, 1998.

[54] Y. Y. Yao. Interval sets and interval-set algebras. In Proceedings of the 8th IEEE International Conference on Cognitive Informatics, pages 307-314, 2009.

[55] Y. Y. Yao, X. Li, T. Y. Lin, and Q. Liu. Representation and classification of rough set models. In T. Y. Lin and A. M. Wildberger, editors, Soft Computing, pages 44-47. Society for Computer Simulation, San Diego, 1995.

[56] L. Zadeh. Fuzzy sets. Information and Control, 8:338-353, 1965.

[57] W. Zakowski. Approximations in the space $(\mathrm{u}, \pi)$. Demonstratio Math., XVI:761-769, 1983. 